\title{
In Reference to "Pilot Study Aiming to Support Sleep Quality and Duration During Hospitalizations"
}

\author{
Nolan R. Machado, BA¹, Samantha L. Anderson, BA², Vineet M. Arora, MD, MAPP²
}


Internal Medicine, Chicago, Illinois.

We commend Gathecha et al. ${ }^{1}$ on the implementation of a well-formed, multicomponent sleep intervention to improve sleep in hospitalized patients. While they were unable to show objective improvement in sleep outcomes, they found improvements in patient-reported sleep outcomes across hospital days, implying that multiple hospital nights are needed to realize the benefits. We wish to propose an alternative strategy. To produce a more observable and immediate improvement in patient sleep outcomes, the behavioral economics principle of nudges ${ }^{2}$ could be an effective way to influence hospital staff toward sleep-promoting practices.

In focus groups at the University of Chicago Medicine, nurses, hospitalists, and residents reported unnecessary nocturnal disruptions were the "default" option hardwired in electronic medical records admission order sets. It was time-consuming to enter orders that minimized unnecessary nocturnal disruptions, such as forgo overnight vitals for stable patients. Given that changing default settings of order sets have been shown to effectively nudge physicians in other areas, ${ }^{3-5}$ altering default settings in admission orders could facilitate physicians' adherence to sleep-promoting practices. An intervention combining these nudges with educational initiatives may be more effective in sustained reductions in nocturnal disruptions and improved inpatient sleep from the start of a hospital stay.

\section{References}

1. Gathecha E, Rios R, Buenaver LF, Landis R, Howell E, Wright S. Pilot study aiming to support sleep quality and duration during hospitalizations. J Hosp Med. 2016;11(7):467-472. doi:10.1002/jhm.2578.

2. Thaler R, Sunstein C. Nudge: Improving Decisions About Health, Wealth and Happiness. New Haven, CT: Yale University Press; 2008.

3. Bourdeaux CP, Davies KJ, Thomas MJC, Bewley JS, Gould TH. Using "nudge" principles for order set design: a before and after evaluation of an electronic prescribing template in critical care. BMJ Qual Saf. 2014;23(5):382-388. doi:10.1136/ bmjqs-2013-002395.

4. Halpern SD, Ubel PA, Asch DA. Harnessing the power of default options to improve health care. N Engl J Med. 2007;357(13):1340-1344. doi:10.1056/NEJMsb071595.

5. Ansher C, Ariely D, Nagler A, Rudd M, Schwartz J, Shah A. Better medicine by default. Med Decis Making. 2014;34(2):147-158. doi:10.1177/0272989X13507339.

\section{The Authors Reply, "Pilot Study Aiming to Support Sleep Quality and Duration During Hospitalizations"}

\author{
Evelyn Gathecha, $\mathrm{MD}^{1}$, Scott Wright, $\mathrm{MD}^{1,2}$
}

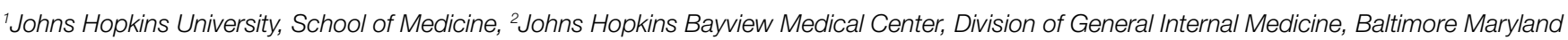

We thank the authors for their comments and thoughts about our recent publication. ${ }^{1}$ Their suggestion that the incorporation of principles from the "Nudge Theory" might enhance the impact of our sleep intervention and shorten the lag time until patients appreciate the benefits is interesting. ${ }^{2}$ Our study aimed to assess the effect of a sleep-promoting intervention on sleep quality and duration among hospitalized patients within a quasi-experimental prospective study design. As is the case at the University of Chicago hospital described in Machado's letter, nocturnal disruptions are also the "default" in order sets in our electronic medical records (EMR). Because the EMR team at our hospital is stretched thin with more requests than it can fulfill, it was not feasible or possible to incorporate any sleep supporting changes when designing the pilot.

Complementing sleep-promoting procedures for hospital- ized patients with "nudge" principles, such as the use of choice architecture with appropriate EMR defaults or even incentives and mappings, seems like a wise recommendation. ${ }^{3}$ Regular nudges may be helpful for sustaining any multicomponent interventions in healthcare delivery that rely on cooperation by multiple parties. It appears as if evidence is growing that "nudge principles" can augment behavior change attributable to interventions. ${ }^{4,5}$ Sleep-promoting nudges, namely "anti-nudges" by members of the healthcare team, should help patients to sleep better during their hospitalizations, when sleep is critically important to recovery and health restitution.

\section{References}

1. Gathecha E, Rios R, Buenaver LF, Landis R, Howell E, Wright S. Pilot study aiming to support sleep quality and duration during hospitalizations. J Hosp Med. 2016;11(7):467-472. doi:10.1002/jhm.2578.

2. Thaler R, Sunstein C. Nudge: Improving Decisions About Health, Wealth and Hap- 
piness. New Haven, CT: Yale University Press; 2008.

3. Bourdeaux CP, Davies KJ, Thomas MJC, Bewley JS, Gould TH. Using "nudge" principles for order set design: a before and after evaluation of an electronic prescribing template in critical care. BMJ Qual Saf. 2014;23(5):382-388. doi:10.1136/ bmjqs-2013-002395

4. Hollands GJ, Shemilt I, Marteau TM, et al. Altering micro-environments to change population health behaviour: towards an evidence base for choice architecture in terventions. BMC Public Health. 2013;13:1218. doi:10.1186/1471-2458-13-1218.
5. Arno A, Thomas S. The efficacy of nudge theory strategies in influencing adult dietary behavior: a systematic review and meta-analysis. BMC Public Health. 2016;16:676. doi:10.1186/s12889-016-3272-x.

Received: August 10, 2016; Accepted: August 11, 2016.

2017 Society of Hospital Medicine DOI 10.1002/jhm.2682 